\title{
Graded Essential Extensions and Graded Injective Modules
}

\author{
Salah EI Din S. Hussein, Essam El Seidy, H. S. Diab* \\ Department of Mathematics, Faculty of Science, Ain Shams University, Abasaya-Cairo, Egypt
}

Email address:

mynsalah@hotmail.com (S. E. D. S. Hussein), esam_elsedy@hotmail.com (E. E. Seidy), hesham_diab88@yahoo.com (H. S. Diab)

\section{To cite this article:}

Salah El Din S. Hussein, Essam El Seidy, H. S. Diab. Graded Essential Extensions and Graded Injective Modules. Pure and Applied Mathematics Journal. Vol. 4, No. 2, 2015, pp. 47-51. doi: 10.11648/j.pamj.20150402.13

\begin{abstract}
In this paper we establish the relation between graded essential extensions of graded modules and injectivity of such modules. We relate the graded injective hull $E_{g r}(M)$ of a graded module $M$ with the graded essential extensions of $M$. We round off by establishing necessary and sufficient conditions for indecomposability of graded injective modules in terms of their graded injective hulls.
\end{abstract}

Keywords: Graded Injective Module, Graded Essential Extension, Graded Injective Hull

\section{Introduction}

The study of projective and injective modules constitutes the backbone of the modern homological algebra. Injective modules are closely related to essential extensions. Actually, if $\mathrm{R}$ is an associative ring with 1 then an $\mathrm{R}$-module $\mathrm{M}$ is injective if and only if it has no proper essential extension. Moreover, the idea of essential extensions has been proved to be indispensable in the formation of the injective hull of a module which is crucial for the theory of rings of quotient. For over forty years, the theory of injective modules has been widely investigated and various aspects of this theory has been completely settled, cf. [1],[2],[3],[4].

This paper is devoted to the study of the interplay between graded essential extensions and graded injective modules. In section 2 we recall some basic notions, definitions, and preliminary results. In section 3 we investigate the relation between the concept of graded essential extensions and injectivity of graded modules. Actually, after deriving the basic properties of graded essential extensions in Proposition 3.1, we provide an important characterization of graded injective modules in terms of graded essential extensions. Graded versions of the well known results of Echmann, Schopf, Baer, Bass, and Papp are proved in Theorem 3.4 and Theorem 3.8. We round off by establishing equivalent conditions for indecomposability of graded injective modules (Theorem 3.11).

\section{Preliminaries}

Throughout this paper $G$ is a multiplicative group with identity e and $R=\underset{\sigma \in G}{\oplus} R_{\sigma}$ is a G-graded ring. The elements of $\bigcup_{\sigma \in G} R_{\sigma}$ are called homogeneous elements of $\mathrm{R}$. All R-modules are left R-modules and by R-mod we denote the category of left R-modules. An R-module $\mathrm{M}$ is said to be G-graded if $M=\underset{\sigma \in G}{\oplus} M_{\sigma}$, for additive subgroups $M_{\sigma}$ of M, satisfying $R_{\sigma} M_{\tau} \subset M_{\sigma \tau}$ for all $\sigma, \tau \in G$.

For any G-graded R-modules $\mathrm{M}$ and $\mathrm{N}$, an R-linear map $f: M \rightarrow N$ is said to be a graded morphism of degree $\tau, \tau \in G$, if $f\left(M_{\sigma}\right) \subset N_{\sigma \tau}$ for all $\sigma \in G$. By R-gr we denote the category of G-graded R-modules and the morphisms are taken to be the graded morphisms of degree e. If $f: M \rightarrow N$ is a graded morphism of degree e we say that $\mathrm{f}$ is a gr-homomorphism. A submodule $\mathrm{N}$ of a G-graded $\mathrm{R}$-module $\mathrm{M}$ is said to be a graded submodule of $\mathrm{M}$ if $N=\underset{\sigma \in G}{\oplus}\left(N \cap M_{\sigma}\right)$, or equivalently, if for any $x \in N$ the homogenous components of $x$ are again in $\mathrm{N}$. By $N \leq_{g r} M$ we denote a graded submodule $\mathrm{N}$ of the graded module M. If $M \in R-g r$ and $K \leq_{g r} M$ then by Zorn's lemma there exist a graded submodule $\mathrm{C}$ of $\mathrm{M}$ which is maximal with respect to $K \cap C=0$. The submodule $\mathrm{C}$ is called a graded complement of $\mathrm{K}$ in $\mathrm{M}$. We say that $M \in R-g r$ is gr-injective if for any gr-monomorphism $\alpha: N \rightarrow M$ and any gr-homomorphism $\beta: N \rightarrow L$, where $N, L \in R-g r$, there exists a gr-homomorphism $\gamma: M \rightarrow L$ such that $\beta=\gamma \alpha$. As in the ungraded one can easily observe that a graded R-module $\mathrm{M}$ is gr-injective if and only if, 
whenever $K \leq_{g r} N$ in R-gr, any gr-homomorphism $f: K \rightarrow M$ can be extended to a gr-homomorphism $f^{\prime}: N \rightarrow M$.

For details on graded rings and modules we refer to [5],[6], while for details on extended injectivities of modules we refer to [7],[8]

Lemma 2.1. (Baer criterion) A graded R-module $M$ is gr-injective if and only if whenever I is a graded left ideal of $\mathrm{R}$, every gr-homomorphism $f: I \rightarrow M$ extends to a gr-homomorphism $f^{\prime}: R \rightarrow M$.

Proof. Similar to the proof of Baer's theorem in the ungraded case, cf. [7] and [8].

Remark 2.2. The extension of $f$ to $f^{\prime}$ in Lemma 2.1 means exactly to find an element $m=f^{\prime}(1) \in M$ such that for every $\quad x \in I, f(x)=f^{\prime}(x)=x . f^{\prime}(1) \quad$ and then $f(x)=x . m$, thus to extend $f$ to $f^{\prime}$ is equivalent to the condition that $f$ is just a right multiplication by an element $m \in M$ (in such case we write $f=\cdot m$ ). Moreover, since $1 \in R_{e} \quad$ and $f^{\prime}$ is graded of degree $\mathrm{e}$ then $f^{\prime}(1)=m \in M_{e} \subset h(M)$.

\section{Graded Essential Extensions and Graded Injectivity}

If $\mathrm{N}$ is a graded submodule of a graded $\mathrm{R}$-module $\mathrm{M}$ then $\mathrm{N}$ is called a gr-essential submodule of $\mathrm{M}$, or $\mathrm{M}$ is called a gr-essential extension of $\mathrm{N}$ if $N \cap X \neq 0$ for every non-zero graded submodule $X$ of $\mathrm{M}$. This state of affairs is denoted by $N \leq_{g r}^{e s s .} M$. Obviously $N \leq_{g r}^{e s s .} M$ if and only if for each $0 \neq x$ in $\mathrm{h}(\mathrm{M})$ there exists an $a \in h(R)$ such that $0 \neq a x \in h(N)$.

In the next proposition we state and prove the graded version of the well known basic properties of essential extensions.

Proposition 3.1. Let $\mathrm{M}$ be a graded R-module

1) If $K \leq_{g r} N \leq_{g r} M$ then $K \leq_{g r}^{e s s .} M$ if and only if $K \leq_{g r}^{e s s} N$ and $N \leq_{g r}^{e s s .} M$.

2) If $K \leq_{g r}^{e s s .} N \leq_{g r} M \& K_{1} \leq_{g r}^{e s s .} N_{1} \leq_{g r} M \quad$ then $K \cap K_{1} \leq_{g r}^{e s s .} N \leq_{g r} N \cap N_{1}$.

3) If $\alpha \in \operatorname{Hom}_{R-g r}(M, N)$ and $K \leq_{g r}^{\text {ess. }} N \leq_{g r} N$, then $\alpha^{-1}(K) \leq_{g r}^{e s s .} M$.

4) Let $M \underset{i \in I}{\oplus} M_{i}$, where $M_{i} \leq_{g r} M$ for each i, and let $K_{i} \leq_{g r} M_{i}$ for each i. Then $\underset{i \in I}{\oplus} K_{i} \leq_{g r}^{e s s .} M$ if and only if $K_{i} \leq_{g r}^{\text {ess. }} M_{i}$ for each i.

5) Let $K \leq_{g r} M$. If $\mathrm{C}$ is a graded complement of $\mathrm{K}$ in $\mathrm{M}$ then (i) $K \oplus C \leq_{g r}^{\text {ess. }} M$

(ii) $(K \oplus C) / C \leq_{g r}^{e s s .} M / C$.

Proof. 1. Suppose that $K \leq_{g r}^{e s s .} N$ and $N \leq_{g r}^{e s s .} M$. If $0 \neq x \in h(M)$ then there exists $a \in h(R)$ such that $0 \neq a x \in h(N)$. But $N \leq_{g r}^{\text {ess. }} M$ implies that $0 \neq$ bax $\in h(M)$, for some $b \in h(R)$. This entails that $K \leq_{g r}^{\text {ess. }} M$.

Conversely, if $K \leq_{g r}^{\text {ess. }} M$ and $0 \neq m \in h(N)$ then there exist $c \in h(R)$ for which $0 \neq c m \in h(K) \subseteq h(N)$. Thus $N \leq_{g r}^{e s s .} M$, since $h(N) \subset h(M)$, obvious.

2. We first observe that $K \cap K_{1} \leq_{g r} N \cap N_{1}$. Indeed,

$$
\begin{aligned}
K \cap K_{1}= & \underset{\sigma \in G}{\oplus}\left[\left(K \cap N \cap M_{\sigma}\right) \cap\left(K_{1} \cap N_{1} \cap M_{\sigma}\right)\right] \\
= & \underset{\sigma \in G}{\oplus}\left(K \cap K_{1}\right) \cap\left[\left(N \cap N_{1}\right) \cap M_{\sigma}\right] \\
& =\underset{\sigma \in G}{\oplus}\left(K \cap K_{1}\right) \cap\left(N \cap N_{1}\right)_{\sigma}
\end{aligned}
$$

Now, if $\quad 0 \neq x \in h\left(N \cap N_{1}\right) \quad$ then $\quad x \in h(N) \quad$ and $x \in h\left(N_{1}\right)$. By assumption, there exist $a \in h(R)$ such that $0 \neq a x \in h(K)$. Since $0 \neq a x \in h\left(N_{1}\right)$ (Note that $x \in h\left(N_{1}\right)$ then $a x \in h\left(N_{1}\right)$ as $N_{1}$ is an R-module) then there exists $b \in h(R)$ such that $0 \neq b a x \in h\left(K_{1}\right)$. Obviously, $0 \neq b a \in h(R)$ and $0 \neq b a x \in h(K)$. Thus, $0 \neq b a x \in h\left(K \cap K_{1}\right)$, or equivalently $K \cap K_{1} \leq_{g r}^{\text {ess. }} N \cap N_{1}$.

3. We first claim that $\alpha^{-1}(K) \leq_{g r} M$. Indeed, if $x=\sum_{i=1}^{n} x_{\sigma_{i}} \in \alpha^{-1}(K), \sigma_{i} \in G \quad$ for $\quad$ each $\mathrm{i}$, then $\alpha(x)=\sum_{i=1}^{n}\left(x_{\sigma_{i}}\right)=\sum_{i=1}^{n}(\alpha(x))_{\sigma_{i}}$. Since $K \leq_{g r} N, \alpha\left(x_{\sigma_{i}}\right)=\alpha(x)_{\sigma_{i}} \in K$ for each $i$. Thus $x_{\sigma_{i}} \in \alpha^{-1}(K)$ for each $i$ and we have done.

Let $0 \neq x \in h(M)$. If $\alpha(x)=0$ then $a \alpha(x)=0 \in K$ for each $a \in h(R)$. It follows that there exists $b \in h(R)$ such that $0 \neq b x \in h\left(\alpha^{-1}(K)\right)$. But if $\alpha(x) \neq 0$ then it is obvious, by hypothesis that there exist $c \in h(R)$ such that $0 \neq c x \in h\left(\alpha^{-1}(K)\right)$. This proves our claim.

4. , 5. It is well known that $\underset{i \in I}{\oplus} K_{i} \leq_{g r} \underset{i \in I}{\oplus} M_{i}$ and $k \oplus C / C \leq_{g r} M / C$, cf.[5]. According to proposition 2.3.5 of [6], the proof of 4. and 5. is similar to the proof of lemma 1.1 and lemma 1.7 of [3].

Lemma 3.2. Every graded R-module $M$ has a maximal graded essential extension.

Proof. Let I be a gr-injective module containing $\mathrm{M}$.

Let $\Lambda=\left\{N_{i}\right\}_{i \in I}$ be a family of graded essential extensions of $\mathrm{M}$ such that $M \leq_{g r}^{\text {ess. }} N_{i} \leq I$, for every $i \in I$. Suppose that $\Lambda$ is linearly ordered by inclusion. Let 
$\Gamma=\left\{N_{j}\right\}_{j \in J}$ be a totally ordered subset of $\Lambda$. For every $0 \neq x \in h\left(\bigcup_{j \in J} N_{j}\right)$ we have $x \in h\left(N_{j_{0}}\right)$ for some $j_{0} \in J$. Since $M \leq_{g r}^{e s s .} N_{j_{0}}$ then there exists $a \in h(R)$ such that $0 \neq a x \in M$, thus $M \leq_{g r}^{e s s .} \bigcup_{j \in J} N_{j}$. Hence $\bigcup_{j \in J} N_{j}$ is an upper bound for $\Gamma$. Thus, by Zorn's lemma, $\Lambda$ has a maximal element $\mathrm{E}$. If $E^{\prime}$ is a graded R-module such that $M \leq_{g r}^{\text {ess. }} E^{\prime}$. If $E \nsubseteq E^{\prime}$ then, by the injectivity of I the inclusion $f: E \rightarrow I$ can be extended to a gr-homomorphism $g: E^{\prime} \rightarrow I$. Obviously, $M \cap \operatorname{Kerg}=0 \quad$, and since $M \leq_{g r}^{\text {ess. }} E^{\prime}$ then $\operatorname{Kerg}=0$. Thus $\mathrm{g}$ is a monomorphism. It follows that $E \varsubsetneqq E^{\prime} \cong g\left(E^{\prime}\right) \leq I$ which contradicts the maximality of $E$ in $\Lambda$. Therefore $E$ is a maximal graded essential extension of $\mathrm{M}$.

Theorem 3.3. Suppose that $\mathrm{G}$ is finite. A graded R-module $\mathrm{M}$ is gr-injective if and only if it has no proper gr-essential extension.

Proof. Let M be gr-injective. Suppose that there exists $E \in R-g r$ such that $M<_{g r}^{e s s .} E$. By the injectivity of M, the inclusion $i: M \rightarrow E$ splits in R-gr. Thus, there exists $N \in R-g r$ such that $E=M \oplus N$, since $M \neq E$ then $N \neq 0$. But $N \cap M=0$ which contradicts our assumption.

Conversely, suppose that $\mathrm{M}$ has no proper gr-essential extension. By the graded version of Theorem 5.5.3 of [7], there exist a gr-injective R-module I such that $M \subseteq I$. Let $\mathrm{S}$ be a graded complement of M in I. Proposition 3.1 entails that $M \cong M \oplus S / S \leq_{g r}^{e s s .} I / S$.

Define $\sigma: M \oplus S / S \rightarrow I$ by $\sigma(x+S)=x$ if $x \in M$. Since I is gr-injective, $\sigma$ extends to a gr-homomorphism $\bar{\sigma}: I / S \rightarrow I$. Since $\operatorname{Ker}(\bar{\sigma}) \cap(M \oplus S) / S=0$ then $(M \oplus S) / S \leq_{g r}^{\text {esS. }} \bar{\sigma}(I / S) \quad$. By assumption, $M=\bar{\sigma}(I / S)=\bar{\sigma}(M \oplus S / S)$ and since $\bar{\sigma}$ is monic we get $I=M \oplus S$. Thus $\mathrm{M}$ is a direct summand of the gr-injective I and hence $\mathrm{M}$ is gr-injective as claimed.

The following is the graded version of the well-known result of Echmann, schopf and Baer, [8], [3].

Theorem 3.4. For modules $M \leq I$ in R-gr, the following are equivalent:

1. I is gr- maximal essential over M.

2. I is gr-injective and gr-essential over M.

3. I is gr-minimal injective over $\mathrm{M}$.

Proof. $1 \Rightarrow 2$. Let $M \leq_{g r}^{\text {eSS } I}$ where I is gr-maximal. If $I \leq_{g r}^{e s s} I_{1}$ then, by 1 . of proposition $3.1, M \leq_{g r}^{e s s} I_{1}$. Thus, by maximality of $\mathrm{I}, I=I_{1}$. Hence I has no proper gr-essential extension. Therefore, Theorem 3.3 entails that I is gr-injective module.

$2 \Rightarrow 3$. Let $I_{1}$ be a gr-injective module such that $M \leq_{g r} I_{1} \leq_{g r} I$. Then $I=I_{1} \oplus N$ for some gr-submodule
$N \leq I$. Since $I_{1} \cap N=0, M \leq_{g r} I_{1}$ then $N \cap M=0$, we must have $N=0$ (since $M \leq_{g r}^{e s s} I_{1}$ ), so $I=I_{1}$.

$2 \Rightarrow 3$. We claim that any graded module $R^{M}$ has a graded maximal essential extension:

Fix a gr-injective module I such that $M \subset I$, and consider any family of gr-essential extensions of $\mathrm{M}$ in I that are linearly ordered by inclusion, Let $\mathrm{P}$ be a chain of this family, it is clear that the union is an upper bound of this chain and the union is also gr-essential over M. By Zorn's Lemma, it follows that we can find a submodule $\mathrm{E}$ maximal with respect to the property that $M \leq_{g r}^{e s s} E \leq_{g r} I$. If I is a gr-minimal injective over M, then there exists a maximal essential extension submodule $\mathrm{E}$ over M such that $M \subset E \subset I$. Using (1) $\Rightarrow$ (2), we know that $\mathrm{E}$ is gr-injective, and by minimality of $\mathrm{I}, \mathrm{E}=\mathrm{I}$, thus $\mathrm{I}$ is gr-maximal essential over M.

Definition 3.5. If the modules $M \leq_{g r} I$ satisfy one (and hence all) of the equivalent properties of Theorem 3.4, we say that $I$ is a gr-injective hull (or gr-injective envelope) of $M$.

As we shall prove in Corollary 3.6 below; the gr-injective hull of $\mathrm{M}$ is unique up to isomorphism. The gr-injective hull of $M$ is denoted by $E_{g r}(M)$.

Corollary 3.6. Any two gr-injective hulls $I, I^{\prime}$ of $\mathrm{M}$ are isomorphic over $\mathrm{M}$; that is there exists a gr-isomorphism $g: I^{\prime} \rightarrow I$ which is the identity of M.

Proof. Let $f: M \rightarrow I, \bar{f}: M \rightarrow I^{\prime}$ are gr-monomorphisms. By injectivity of I, $f: M \rightarrow I$ extends to $g: I^{\prime} \rightarrow I$ and we have $\mathrm{g}$ is gr-monomorphism because $\operatorname{ker}(g) \cap \bar{f}(M)=\operatorname{ker}(f)=0$ and $\bar{f}(M) \leq_{g r}^{e s S} I^{\prime}$ then $\operatorname{ker}(g)=0$ therefore $g\left(I^{\prime}\right)$ is a direct summand of I and since every direct summand of gr-injective module is gr-injective then $g\left(I^{\prime}\right)$ is gr-injective containing M, By (3) in Theorem 3.4 we have $g\left(I^{\prime}\right)=I$ and so $\mathrm{g}$ is surjective and hence gr-isomorphism.

Corollary 3.7. 1. If I is gr-injective module containing M, then I contains a copy of $E_{g r}(M)$.

2. If $M \leq_{g r}^{e S S} N$, then $\mathrm{N}$ can be enlarged into a copy of $E_{g r}(M)$ in fact $E_{g r}(N)=E_{g r}(M)$.

Proof.

1. If $M \leq_{g r}^{e s S} I$ such that $I$ is a gr-injective module then $E_{g r}(M) \subseteq I$ because $E_{g r}(M)$ is a minimal gr-injective over M.

2. $N \leq_{g r}^{e s s} E_{g r}(N)$ and $M \leq_{g r}^{e s s} N$ then by 1 . Of Proposition 3.1 we have $M \leq_{g r}^{e S S} E_{g r}(N)$. On the other hand, $M \leq_{g r}^{e s s} E_{g r}(M)$ and $E_{g r}(M)$ is unique up to isomorphism. Hence $E_{g r}(M)=E_{g r}(N)$.

In the following Theorem we prove the graded version of the well known result of Bass and Papp Theorem [9]

Theorem 3.8. For a graded ring $R,{ }_{R} R$ is gr-noetherian if and only if every direct sum of gr-injective left R-module is 
gr-injective.

Proof. Let $Q:=\underset{i \in I}{\oplus} Q_{i}$ be an internal or external direct sum of gr-injective left R-modules $Q_{i}$. By Baer's Criterion, for every left gr-ideal $U \leq R$ and every gr-homomorphism $\rho: U \rightarrow Q$ there exists a gr-homomorphism $\tau: R \rightarrow Q$ with $\rho=\tau i$ where $i: U \rightarrow R$ is the inclusion mapping. Since ${ }_{R} R$ is gr-noetherian, $\mathrm{U}$ is finitely generated:

$$
u=\sum_{i=1}^{n} R u_{i}
$$

The images $\rho\left(u_{i}\right), i=1,2,3, \ldots, n$ of the $u_{i}$ under $\rho$ have components different from zero for only finitely many of the $Q_{i}$, say for the $Q_{i}$ with $i \in I_{0}$ where $I_{0}$ is a finite subset of I. Let $i_{\circ}: \underset{i \in I_{\circ}}{\oplus} Q_{i} \rightarrow \underset{i \in I}{\oplus} Q_{i}$ be the inclusion mapping and let $\rho_{\circ}$ be the gr-homomorphism, induced by the restriction of the domain of $\rho$ to $\underset{i \in I_{\circ}}{\oplus} Q_{i}$ then we have $\rho=i_{\circ} \rho_{\circ}$. Since $I_{\circ}$ is finite, $\underset{i \in I_{\circ}}{\oplus} Q_{i}$ is gr-injective and there exists a gr-homomorphism $\tau_{\circ}$ so that the following diagram is commutative:

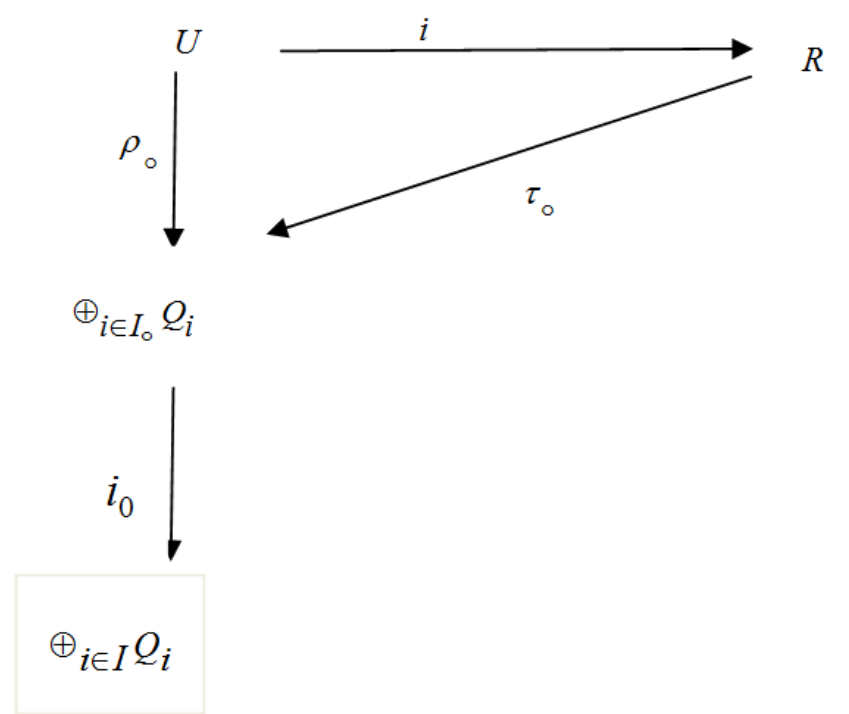

Consequently we have $\rho=i_{\circ} \rho_{\circ}=i_{\circ} \tau_{\circ} i=\tau i$ if we put $\tau:=i_{0} \tau_{\circ}$.

Conversely, consider an arbitrary chain of left gr-ideals

$$
A_{1} \leq A_{2} \leq A_{3} \leq \ldots
$$

And let

$$
A:=\bigcup_{i=1}^{\infty} A_{i}
$$

Now let $\eta_{i}$ be the inclusion mappings

$$
\eta_{i}: A / A_{i} \rightarrow I\left(A / A_{i}\right)
$$

And let

$$
\alpha: A \rightarrow \underset{i=1}{\oplus} I\left(A / A_{i}\right)
$$

be defined by

$$
\alpha(a):=\sum_{i=1}^{n_{a}}\left(a+A_{i}\right) \quad a \in A
$$

Then follows that $\eta_{i}=0$ for $i \geq n$ and consequently $A=A_{i}$ for $i \geq n$

Theorem 3.9. Let $\mathrm{R}$ be a graded noetherian ring, then any graded injective $\mathrm{R}$ - module is a direct sum of indecomposable graded injective submodules.

Proof. First we prove that any gr-injective module contains gr-injective indecomposable submodule. Let $I=E_{g r}(R x), x \in h(I)$.

Since $\mathrm{R}$ is gr-noetherian then it is easily checked that $E_{g r}(R x)$ contains an indecomposable gr-injective submodule. Now, for any gr-injective module $R^{M}$, consider all the families of all gr -injective indecomposable submodules of $\mathrm{M}$ whose sum is direct. By Zorn's lemma there exists a family $\left\{M_{i}: i \in I\right\}$ which is maximal, then $M=N \oplus\left(\oplus_{i} M_{i}\right)$ for some $N \leq_{g r} M$. Since $\oplus_{i} M_{i}$ is gr-injective by Theorem 3.8 and $\mathrm{E}$ is a direct summand of gr-injective as well, then by the first part of the proof E must contian an indecomposable gr-injective submodule which contradicts the maximality of $\left\{M_{i}: i \in I\right\}$, thus $\mathrm{E}$ must be zero. This proves our claim.

Corollary 3.10. Let $\mathrm{N}$ be a finitely generated module over a graded noetherian ring R. Then $E_{g r}(N)$ is a finite direct sum of indecomposable gr-injective submodules.

Proof. By Theorem 3.9, $E_{g r}(N)=\oplus_{i} M_{i}$ where the $M_{i}^{\prime} s$ are indecomposable gr-injective. Since $\mathrm{N}$ is finitely generated, we have $N \subseteq M_{i_{1}} \oplus \ldots \ldots . . \oplus M_{i_{n}}$ for suitable $i_{1}, \ldots \ldots . i_{n}$. By Theorem 3.7, this finite direct sum is gr-injective. Therefore, by Corollary 3.7, we must have $E_{g r}(N)=M_{i_{1}} \oplus \ldots \ldots . . \oplus M_{i_{n}}$.

A nonzero graded module $\mathrm{M}$ is called graded uniform if any nonzero graded submodules of $\mathrm{M}$ intersect nontrivially (equivalently any nonzero graded submodule of $\mathrm{M}$ is indecomposable). A graded ideal $I \leq_{g r} R$ is called graded meet-irreducible if the cyclic graded module $R^{(R / I)}$ is uniform.

Theorem 3.11. For any graded injective module $\mathrm{M}$ over a graded ring $\mathrm{R}$, the following conditions are equivalent:

1. $\mathrm{M}$ is gr- indecomposable.

2. $M \neq 0$, and $M=E_{g r}\left(M^{\prime}\right)$ for any non zero graded submodule $M^{\prime} \leq M$.

3. $\mathrm{M}$ is graded uniform. 
4. $M=E_{g r}(U)$ for some graded uniform module $\mathrm{U}$.

5. $M=E_{g r}(R / u)$ for some graded meet-irreducible ideal $u \leq_{g r} R$.

6. $\mathrm{M}$ is strongly indecomposable, that is, $\operatorname{End}_{g r}(M)$ is a local graded ring.

Proof. 1) $\Rightarrow$ 2) Direct consequence of Theorem 3.9.

2) $\Rightarrow 3$ ) Clear, from the definition of graded uniform module .

3) $\Rightarrow$ 4) Obvious.

$4) \Rightarrow 5$ ) Let $V \neq 0$ be a cyclic submodule of $U$. Since $U$ is graded uniform, $V \leq_{g r}^{\text {ess. }} U$. But then $V \leq_{g r}^{\text {ess. }} E_{g r}(U)$ by 1 . of Proposition 3.1, and this gives $M=E_{g r}(V)$. We are done by identifying $V$ with $R / u$ for a (necessarily meet-irreducible) right ideal $u \leq_{g r} R$.

5) $\Rightarrow 6)$ Here $M=E_{g r}(U)$ where $U=R / u$ is uniform. If $\alpha$ is a nonunit in $E=\operatorname{End}_{g r}\left({ }_{R} M\right)$, then $\operatorname{ker} \alpha \neq 0$. (If ker $\alpha=0$, then $\operatorname{im}(\alpha) \leq_{g r} M$. But $\operatorname{im}(\alpha) \cong M$ injective, so $M=i m(\alpha) \oplus A$ for some $A \neq 0$. Both $i m(\alpha)$ and $\mathrm{A}$ must intersect $\mathrm{U}$ nontrivially, contradicting the fact that $\mathrm{U}$ is uniform.) Therefore, $U \cap \operatorname{ker} \alpha \neq 0$. If $\beta$ is another nonunit in $\mathrm{E}$, then likewise $U \cap \operatorname{ker} \beta \neq 0$ and we have

$$
\operatorname{ker}(\alpha+\beta) \supseteq(U \cap \operatorname{ker} \alpha) \cap(U \cap \operatorname{ker} \beta) \neq 0 .
$$

This implies that $\alpha+\beta$ is a nonunit in $\mathrm{E}$; hence $\mathrm{E}$ is a local ring.

$6) \Rightarrow 1$ ) If $E$ is a local ring, it does not have nontrivial idempotents

\section{Conclusion}

Along the proofs of the results of this paper one can easily discover that the iterated use of essential extensions of graded modules seems to deserve further development. For instance, using Theorem 3.11, it is quite possible to prove the graded version of the important well known theorem of Matlis which states that : If $\mathrm{R}$ is either a commutative noetherian ring or a right artinian ring then there exists a bijection between the set of isomorphism classes of right indecomposable injective $\mathrm{R}$-modules and the set of all prime ideals of $\mathrm{R}$.On the other hand, one can also try to compute the graded injective hull of cyclic non-free graded modules and graded modules over graded noetherian rings which are not PID.Finally, the results of this paper enable us to study the graded extended injectivities of graded modules such as Soc-injectivity, Rad-injectivity, and almost injectivity, cf. [1],[2],[4]. However this is not the aim of this paper, it will be the topic of some forthcoming work.

\section{References}

[1] Amin I., Yousif M., Zeyada N., Soc-injective rings and modules, Comm. in Alg., Vol. 33,No.1, (2005), 4229-4250.

[2] Zeyada Nasr A., Hussein Salah El Din S., Amin Amr K., Rad-injective and almost-injective modules and rings, Alg. Colloquium, Vol.18, No. 3, (2011), 411-418.

[3] Nicholson W.K., Yousif M.F., Quasi-Frobenius rings, Cambridge Tracts in Math. 158, Cambridge Univ. Press, Cambridge, UK, (2003).

[4] Yousif M. F., Zhou Y., Zeyada N., On peseudo-Frobenius rings, Can. Math. Bull., Vol.48 No. 2, (2005), 317-320.

[5] Nastasescu C., Van Oystaeyen F., Graded ring theory, Math. Library, North Holland, Amsterdam 28, (1982).

[6] Nastasescu C., Van Oystaeyen F., Methods of graded rings, Springer-Verlag, Berlin Heidelberg, LNM.1836, (2004).

[7] Kasch F., Modules and rings, Academic Press, New York, L.M.S. Monograph No.17, (1982).

[8] Lam T. Y., Lectures on modules and rings, Graduate Texts in Mathematics, Springer-Verlag, New York, Vol. 189, (1998).

[9] Papp, Zolt'an, On algebraically closed modules, Publicationes Mathematicae Debrecen, Vol.6, (1959), 311-327. 